\title{
NEW LOCALITIES OF ENDEMIC AQUILEGIA KITAIBELII SCHOTT AND CARDAMINOPSIS CROATICA (SCHOTT, NYMAN ET KOTSCHY) JÁV. IN CROATIA
}

\section{Dario Kremer ${ }^{1 *}$, Gordan Lukač ${ }^{2}$, Ana Brkljačić 3 , Joso Brajković ${ }^{4}$, KreŠimir Čulinović ${ }^{5}$ \& Marko Randić}

\author{
${ }^{1}$ Faculty of Pharmacy and Biochemistry, University of Zagreb, Schrottova 39, \\ HR-10000 Zagreb, Croatia \\ ${ }^{2}$ Public Institution "Paklenica National Park", F. Tuđmana 14A, \\ HR-23244 Starigrad Paklenica, Croatia \\ ${ }^{3}$ Public Institution "Velebit Nature Park", Kaniža Gospićka 4b, HR-53000 Gospić, Croatia \\ ${ }^{4}$ Croatian Chamber of Commerce, Chamber of Commerce Otočac, Kralja Zvonimira 16, \\ HR-53220 Otočac
}

5Public institution " Plitvice Lakes National Park", Scientific Research Center "Dr. Ivo Pevalek", HR-53231 Plitvička jezera, Croatia

${ }^{6}$ Public Institution “Priroda”, Grivica 4, HR-51000 Rijeka, Croatia

Kremer, D., Lukač, G., Brkljačić, A., Brajković, J., Čulinović, K. \& Randić, M.: New localities of endemic Aquilegia kitaibelii Schott and Cardaminopsis croatica (Schott, Nyman et Kotschy) Jáv. in Croatia. Nat. Croat., Vol. 24, No. 2., 345-359, Zagreb, 2015.

New localities of two endemic, rare and floristically interesting species in the north-western Dinaric Mountains in Croatia are reported in the paper. Both species are statutorily strictly protected species in Croatia. Aquilegia kitaibelii Schott, as a rare, endemic species, is included in Appendix II of the Habitats Directive (NATURA 2000 Species) and in the IUCN list as a data deficient (DD) species. New localities of A. kitaibelii were found on Mt Obruč and the central and south sections of Mt Velebit, while new localities of Cardaminopsis croatica (Schott, Nyman et Kotschy) Jáv. were discovered on hills in the Gacka Region and the central section of Mt Velebit. In general, both species grow mainly from fissures on rocks together with other petrophilous plant species. It was also noted that $A$. kitaibelii grows on screes. In Gacka Region C. croatica grows in thermophilous habitats, that is, on rocks with different exposures, together with thermophilous sub-Mediterranean species and with some mountain species of common beech and silver fir forests. In the central section of Mt Velebit, C. croatica also grows in a thermophilous community of forest rocks as well as in anthropogenic habitats, like the edges of gravel roads. These observations suggest the ecological adaptability of this endemic species.

Key words: Aquilegia kitaibelii, Cardaminopsis croatica, endemic species, NATURA 2000 species, Dinaric Mountains

Kremer, D., Lukač, G., Brkljačić, A., Brajković, J., Čulinović, K. \& Randić, M.: Novi lokaliteti endemičnih vrsta Aquilegia kitaibelii Schott i Cardaminopsis croatica (Schott, Nyman et Kotschy) Jáv. in Hrvatskoj. Nat. Croat., Vol. 24, No. 2., 345-359, Zagreb, 2015.

U radu su navedeni novi, do sada neobjavljeni lokaliteti dviju endemičnih i floristički zanimljivih vrsta rasprostranjenih na području sjeverozapadnih Dinarida u Hrvatskoj. Obje vrste su u nas i zakonom strogo zaštićene. Jedna od njih je Kitaibelov pakujac (Aquilegia kitaibelii Schott) koji je kao rijetka, endemična vrsta uvrštena na popis NATURA 2000 vrsta. Nova nalazišta Kitaibelova pakujaca zabilje-

\footnotetext{
* corresponding author; e-mail: dkremer@pharma.hr
} 
žena su na širem području planinske skupine planine Obruč te na srednjem i južnom Velebitu, dok su novi lokaliteti hrvatske gušarke (Cardaminopsis croatica (Schott, Nyman et Kotschy) Jáv.) utvrđeni na području srednjeg Velebita i na padinama brežuljaka regije Gacke. Obje vrste na većini novih nalazišta rastu u pukotinama stijena zajedno s drugim petrofilnim vrstama, a Kitaibelov pakujac zabilježili smo i na točilima. Za hrvatsku gušarku posebno su zanimljiva nalazišta u regiji Gackoj, gdje raste na termofilnim stijenama različitih ekspozicija, u okruženju mješavine termofilnih submediteranskih vrsta i nekih brdskih vrsta bukovo-jelovih šuma. Uz Premužićevu stazu na srednjem Velebitu C. croatica se također pojavljuje u termofilnim zajednicama šumskih stijena. Opaženo je i da, osim stijena, hrvatska gušarka nastanjuje i sekundarna, antropogena staništa, kao što su rubovi šumskih cesta, što ukazuje na ekološku plastičnost ove endemične vrste.

Ključne riječi: Aquilegia kitaibelii, Cardaminopsis croatica, endemične vrste, NATURA 2000 vrste, Dinarske planine

\section{INTRODUCTION}

Aquilegia kitaibelii Schott (Ranunculaceae) is a perennial plant up to $40 \mathrm{~cm}$ tall and with 1-2 (-6) blue-violet flowers. It is distributed in Croatia and Bosnia and Herzegovina at altitudes ranging from 1200 to $1700 \mathrm{~m}$ a.s.l. (Cullen \& Heywood, 1964; Kremer, 2008). Additionally, there are unconfirmed observations that $A$. kitaibelii grows on Mt Snežnik in Slovenia (Роровлıк et al., 2013). The literature data about the presence of $A$. kitaibelii in northeastern Italy (ŠILIĆ, 1990) also needs to be confirmed because it is possible that A. kitaibelii of northeastern Italy is closely related to the species Aquilegia bertolonii Schott s.l. (Podobnik et al., 2013). In Croatia it can be found on Mt Obruč, Mt Risnjak, Mt Snježnik, Bijele and Samarske stijene, Mt Velebit, Mt Plješivica, Mt Dinara, Mt Biokovo and in Plitvice Lakes National Park (Degen, 1938: 118; Horvat, 1962; Kušan, 1969; Horvat et al., 1974; ForenbaCher, 1990; ŠEgulja, 1994; ŠEgulja et al., 1994; Alegro, 2004; RANDić, 2010; Niкоцić (ed.), 2013). Aquilegia kitaibelii is a statutorily strictly protected species in Croatia (Anonymous, 2013). As a rare, endemic species A. kitaibelii is included in Appendix II of the Habitats Directive (NATURA 2000 Species). The species is recognized in the IUCN list, though as DD (data deficient), so it is of importance to collect as many data as possible about distribution range, number of populations, number of plants per population, and habitat conditions. Aquilegia kitaibelii is also included in Appendix IV of Council Directive 92/43/EEC of 21 May 1992 on the conservation of natural habitats and of wild fauna and flora (Anonymous, 2013). It is also included in Appendix I of the Bern Convention on the Conservation of European Wildlife and Natural Habitats as strictly protected flora species. Additionally, it is worth mentioning that Aquilegia species are a well-known plant model organism in the field of evolutionary biology (FIOR et al., 2013).

Cardaminopsis croatica (Schott, Nyman et Kotschy) Jáv. (syn. Arabidopsis croatica (Schott ex Nyman et Kotschy) O'Kane et Al-Shehbaz, Arabis croatica Schott, Nyman et Kotschy, Brassicaceae) is a perennial plant up to $20 \mathrm{~cm}$ tall, with a glabrous, erect, flexuous, divaricately branched stem and with several white or pinkish flowers. It is a montane to subalpine plant distributed in Croatia and Bosnia and Herzegovina within an altitude ranging from 500 to $1500 \mathrm{~m}$ a.s.l. (ŠILIĆ, 1990; SснміскL et al., 2012). In Croatia, it can be found on mountainous to subalpine parts of Mt Velika Kapela, Mt Senjsko bilo, Mt Velebit, and Mt Lička Plješivica. Also, it has been found at lower altitudes at some localities in Lika (Degen, 1938; Horvat, 1962; Horvat et al., 1974; Forenbacher, 1990; ŠEgulja, 1994; Alegro, 2004; Nikolić (ed.), 2014). According to the new taxonomic point of view, C. croatica was removed from the genus Cardaminopsis and placed in the genus Arabidopsis (AL-Shehbaz and O'KANE, 2002). Being closely related to the preeminent plant model 
organism Arabidopsis thaliana (L.) Heynh., C. croatica has become a very interesting species (SchmickL, 2012; Hohmann et al., 2014). This is yet another reason why it is important to find as many localities of $C$. croatica as possible and reveal any differences there might be in the ecological characteristics among habitats of this endemic species. Additionally, Cardaminopsis croatica is a statutorily strictly protected species in Croatia (AnOnymous, 2013).

The aim of this paper is to present new localities of the endemic Illyrian-Balkan species A. kitaibelii and C. croatica in Croatia. Any information concerning the localities of A. kitaibelii and C. croatica helps to broaden our knowledge of their population numbers, environmental conditions, and range. Such information could help in putting in place monitoring schemes for both species, developing plans for managing protected areas and ecological networks, and ensuring in situ and ex situ conservation. Additionally, the data are important for making re-evaluations of the NATURA 2000-documents and the Croatian Red Book of Vascular Plants (KREMER et al., 2014).

\section{MATERIALS AND METHODS}

The new localities were discovered during several field trips undertaken from June 2007 to October 2014. Each locality was described via data on altitude and location obtained using Garmin eTrex Vista HCx and Gauss-Krüger coordinates system. Standard keys for identification were used (Cullen \& Heywood, 1964; ŠIlić, 1990; DomAC, 1994). Nikolić (ed.) (2014) was employed as a standard for the nomenclature of the species. A list of prevailing species which grow inside and on the border of the investigated populations of A. kitaibelii and C. croatica was added. Habitat type was determined according to the National Habitat Classification (Anonymous, 2009).

Voucher specimens were deposited in the Fran Kušan Herbarium at the Department of Pharmaceutical Botany with the Fran Kušan Pharmaceutical Botanical Garden of the Faculty of Pharmacy and Biochemistry, University of Zagreb, Croatia.

\section{RESULTS AND DISCUSSION}

Tab. 1 displays a total of 39 new localities of A. kitaibelii and C. croatica in the northwestern Dinaric Mountains in Croatia.

\section{Localities of Aquilegia kitaibelii}

New localities of $A$. kitaibelii were found in the hinterland mountains of the city of Rijeka and on Mt Velebit. Horvat (1962) mentioned the presence of A. kitaibelii on Mt Risnjak and Mt Obruč, but without specific information about where it grows. SurINA (2013) also described two localities of A. kitaibelii on Mt Obruč. The presence of C. croatica on Mt Risnjak was not confirmed during these investigations although it was noted by ŠEGULJA et al. (1994).

\section{Pakleno (Mt Obruč)}

Several dozen plants of $A$. kitaibelii were found on the north-exposed, partly shaded calcareous rocks at the bottom of Mt Obruč in the Pakleno area. Nearby vegetation is represented by subalpine underwoods and forests of Abies alba Mill. developed on stone blocks. Aquilegia kitaibelii grows from the fissures in limestone together with Asplenium fissum Kit., A. ruta-muraria L., Galium lucidum All., Cyclamen purpurascens Mill., Gentiana 


\begin{tabular}{|c|c|c|c|c|c|c|c|c|c|c|c|c|c|c|c|c|c|c|c|c|}
\hline 总 & 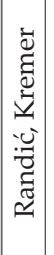 & 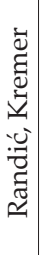 & 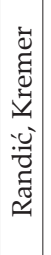 & 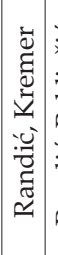 & 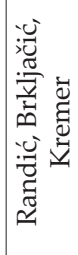 & 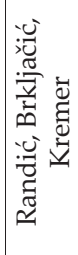 & 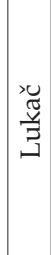 & $\begin{array}{l}2 \\
\widetilde{\tilde{g}} \\
3 \\
3\end{array}$ & 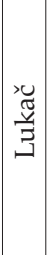 & 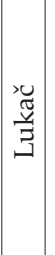 & 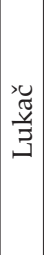 & 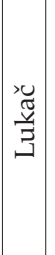 & \begin{tabular}{l|l}
$\underset{\widetilde{g}}{3}$ & $>$ \\
3 &
\end{tabular} & & 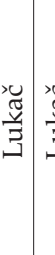 & 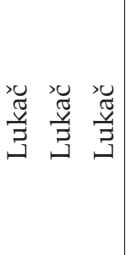 & 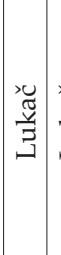 & 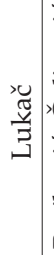 & 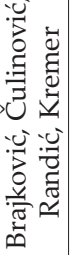 & 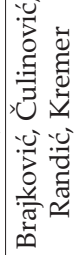 \\
\hline صّ & 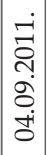 & 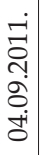 & 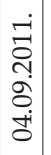 & $\begin{array}{l}\dot{\vec{g}} \\
\overrightarrow{0} \\
\tilde{a} \\
\dot{\delta} \\
\dot{\delta}\end{array}$ & 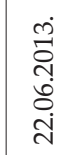 & 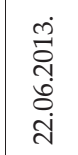 & 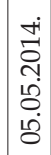 & 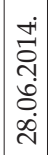 & 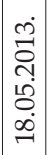 & 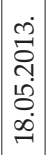 & 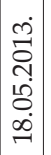 & 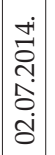 & 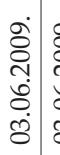 & 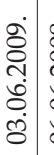 & 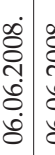 & 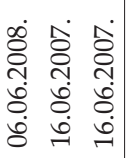 & 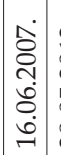 & 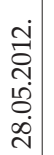 & 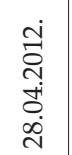 & 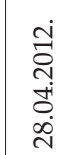 \\
\hline 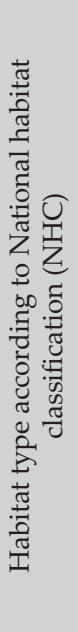 & 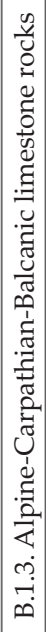 & 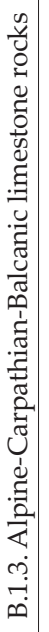 & 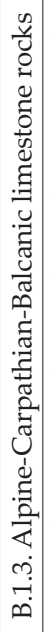 & 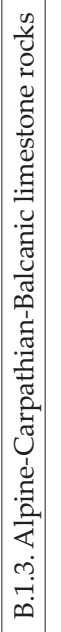 & 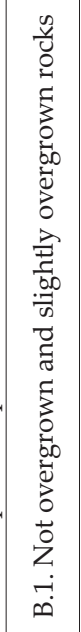 & 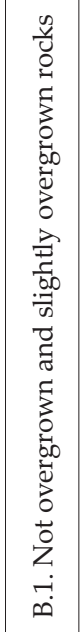 & 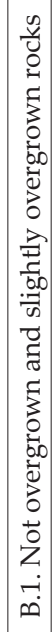 & 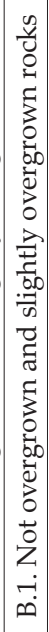 & 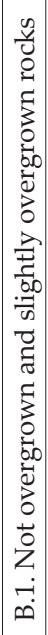 & 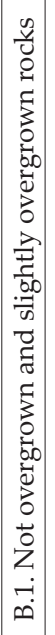 & 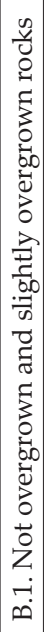 & 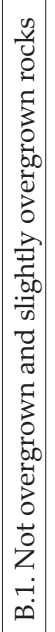 & 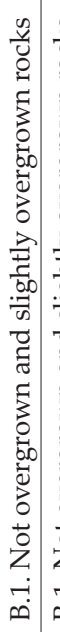 & 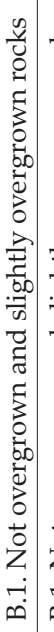 & 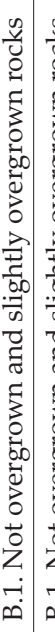 & 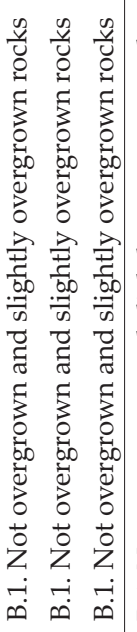 & 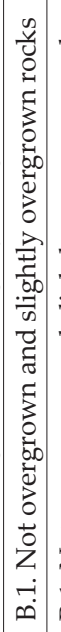 & 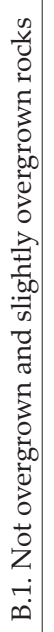 & 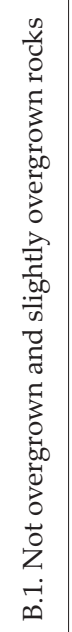 & 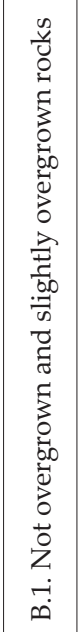 \\
\hline 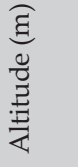 & $\vec{\sigma}$ & $\stackrel{\substack{\infty \\
=}}{=}$ & 芯 & $\begin{array}{l}\vec{m} \\
\stackrel{\oplus}{\sim}\end{array}$ & $\begin{array}{l}\infty \\
\stackrel{\infty}{2}\end{array}$ & $\begin{array}{l}\text { D } \\
\stackrel{\text { I }}{二}\end{array}$ & $\stackrel{m}{\sigma}$ & 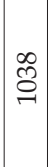 & 芯 & $\begin{array}{l}\infty \\
\swarrow \\
\end{array}$ & బิ & $\mid \begin{array}{l}+ \\
\stackrel{1}{0} \\
0\end{array}$ & $\stackrel{\overbrace{}}{0}$ & $\underset{\sigma}{\tilde{\sigma}}$ & \begin{tabular}{l}
1 \\
\multirow{2}{*}{}
\end{tabular} & 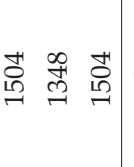 & 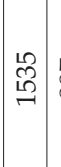 & ڤે & के & 욤 \\
\hline 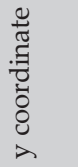 & 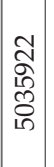 & 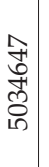 & 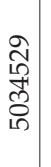 & $\begin{array}{l}0 \\
10 \\
0 \\
0 \\
0 \\
10\end{array}$ & 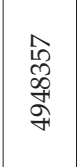 & 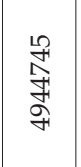 & $\begin{array}{l}F \\
\text { 孛 } \\
\text { 采 }\end{array}$ & $\begin{array}{l}2 \\
\overrightarrow{2} \\
\vec{z} \\
\frac{7}{q}\end{array}$ & 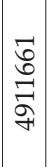 & 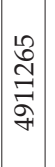 & 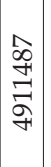 & 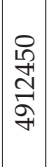 & 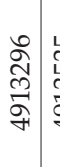 & 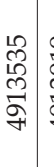 & 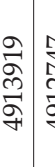 & 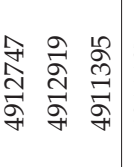 & $\mid$\begin{tabular}{c}
0 \\
0 \\
$\vec{G}$ \\
$\vec{\sigma}$ \\
\multirow{q}{*}{}
\end{tabular} & 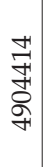 & 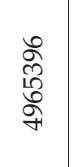 & 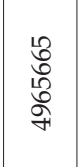 \\
\hline 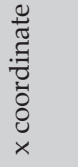 & $\mid \begin{array}{c}1 \\
\vdots \\
0 \\
1 \\
04 \\
10\end{array}$ & $\begin{array}{l}8 \\
0 \\
0 \\
10 \\
7 \\
10\end{array}$ & 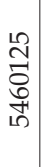 & 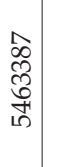 & $\begin{array}{l}\text { oి } \\
\text { ò } \\
\text { के }\end{array}$ & 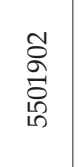 & $\begin{array}{c}0 \\
0 \\
N \\
0 \\
10 \\
10 \\
10\end{array}$ & $\begin{array}{ll}\overrightarrow{5} \\
0+1 \\
0 \\
0 \\
10 \\
10\end{array}$ & $\begin{array}{l}0 \\
0 \\
\infty \\
0 \\
10 \\
10 \\
10\end{array}$ & 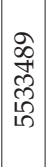 & 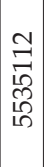 & 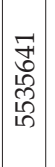 & 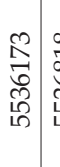 & 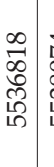 & 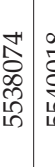 & 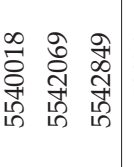 & 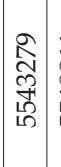 & $\begin{array}{l}\vec{F} \\
\infty \\
\infty \\
10 \\
10\end{array}$ & $\begin{array}{l}\vec{\sigma} \\
\stackrel{\infty}{0} \\
\stackrel{0}{20} \\
\llcorner n\end{array}$ & 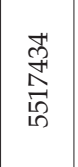 \\
\hline 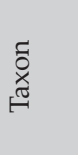 & $\mid$ & 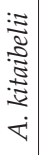 & 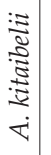 & 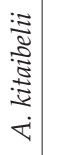 & 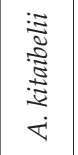 & 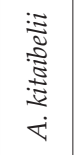 & 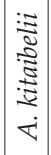 & 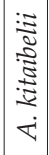 & 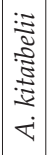 & 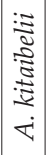 & $\mid$ & 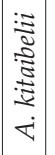 & 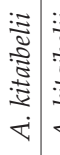 & 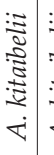 & 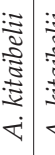 & 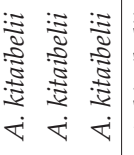 & 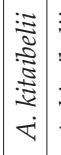 & 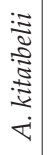 & 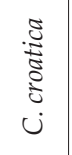 & 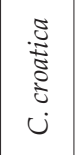 \\
\hline$\dot{\circ}$ & -1 & $N$ & $\infty$ & + & 10 & 0 & 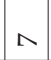 & $\infty$ & $a$ & 인 & $\exists$ & $\cong$ & $\stackrel{M}{\sim}$ & $\exists !$ & $\stackrel{2}{\rightarrow} \div$ & $\stackrel{0}{\rightleftharpoons} \stackrel{\infty}{\sim}$ & $\stackrel{2}{-}$ & 요 & $\vec{N}$ & N \\
\hline
\end{tabular}




\begin{tabular}{|c|c|c|c|c|c|c|c|c|c|c|c|c|c|c|c|c|}
\hline 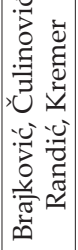 & 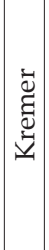 & 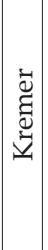 & 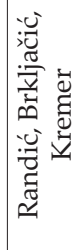 & 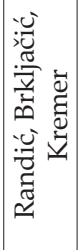 & 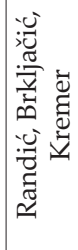 & 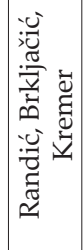 & 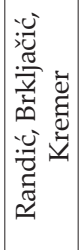 & 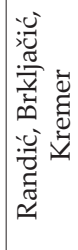 & 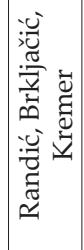 & 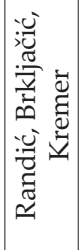 & 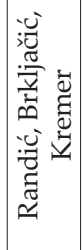 & 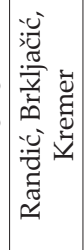 & 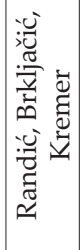 & 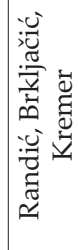 & 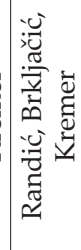 & 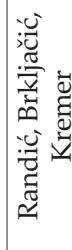 \\
\hline 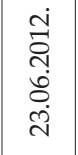 & 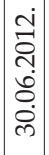 & 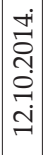 & 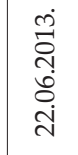 & 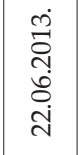 & 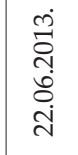 & 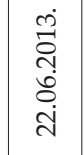 & 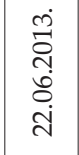 & 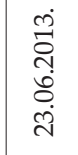 & 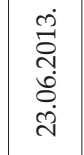 & 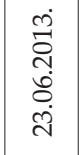 & 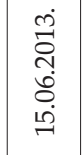 & 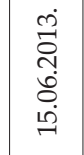 & 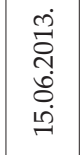 & 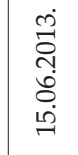 & 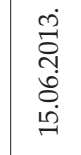 & 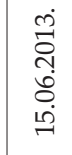 \\
\hline 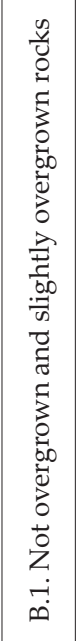 & 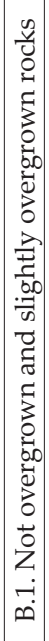 & 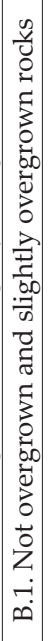 & 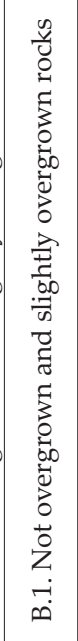 & 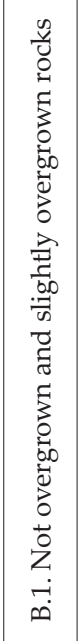 & 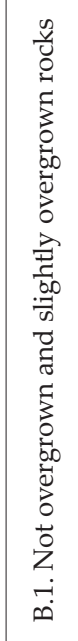 & 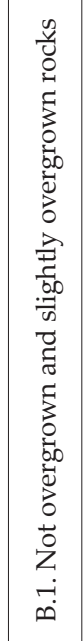 & 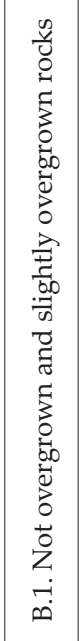 & 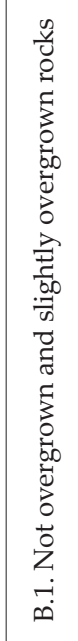 & 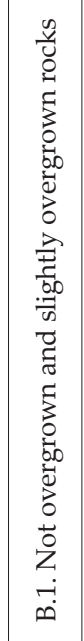 & 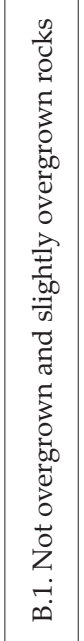 & 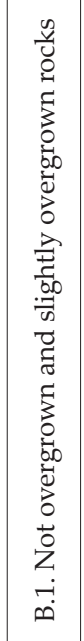 & 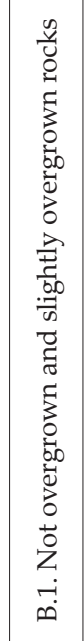 & 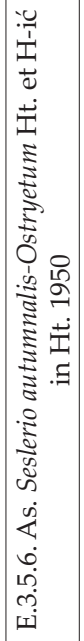 & 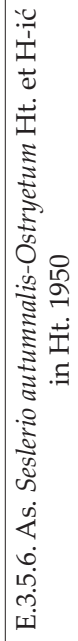 & 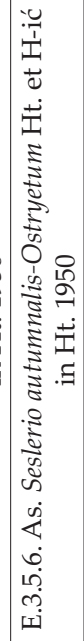 & 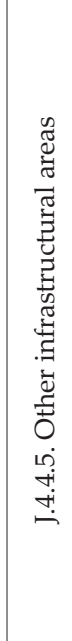 \\
\hline டீ & 志 & $\begin{array}{l}\mathbf{8} \\
\text { co } \\
-1\end{array}$ & $\stackrel{\vec{m}}{\vec{m}}$ & ঐे & $\begin{array}{l}\infty \\
\stackrel{\infty}{\sigma} \\
\stackrel{0}{n}\end{array}$ & $\begin{array}{l}\text { 고 } \\
\stackrel{n}{\sim}\end{array}$ & $\begin{array}{l}\text { \& } \\
\stackrel{1}{\simeq}\end{array}$ & తิ & $\stackrel{-\infty}{\stackrel{\circ}{\circ}}$ & ลૂ & సิ & \& & 离 & హి & న్ & $\stackrel{\infty}{\circ}$ \\
\hline \begin{tabular}{l}
$\infty$ \\
$\stackrel{0}{0}$ \\
$\stackrel{0}{+}$ \\
\multirow{+}{*}{}
\end{tabular} & 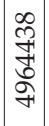 & 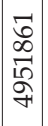 & $\begin{array}{l}\infty \\
\infty \\
\circ \\
\stackrel{+}{+}\end{array}$ & $\begin{array}{l}\stackrel{\infty}{\Lambda} \\
\vec{\infty} \\
\stackrel{+}{+}\end{array}$ & 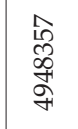 & 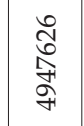 & 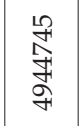 & \begin{tabular}{l}
$\stackrel{2}{\&}$ \\
\multirow{2}{*}{} \\
f
\end{tabular} & 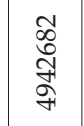 & $\begin{array}{l}\stackrel{1}{\hat{~}} \\
\widehat{\sigma} \\
\text { హे }\end{array}$ & 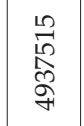 & $\begin{array}{l}\bar{\sigma} \\
\infty \\
\check{\sigma} \\
\sigma\end{array}$ & 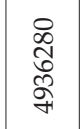 & 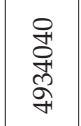 & 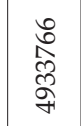 & $\begin{array}{l}\stackrel{0}{\infty} \\
\text { ర్ } \\
\sigma\end{array}$ \\
\hline $\begin{array}{l}\text { के } \\
\text { ఫु } \\
\text { Nิ } \\
\text { ம் }\end{array}$ & $\begin{array}{l}0 \\
\hat{2} \\
\infty \\
10 \\
10 \\
10 \\
\end{array}$ & 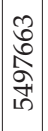 & $\begin{array}{l}\text { సे } \\
\stackrel{\infty}{\circ} \\
\text { जै }\end{array}$ & 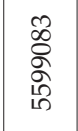 & $\begin{array}{l}\text { ஜి } \\
\text { ळू } \\
\text { চै }\end{array}$ & $\begin{array}{l}\text { ळ } \\
\text { ळू } \\
\text { ळे }\end{array}$ & 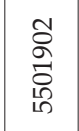 & 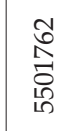 & 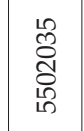 & 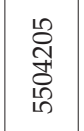 & 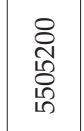 & 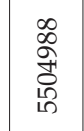 & $\begin{array}{l}\infty \\
2 \\
\infty \\
0 \\
0 \\
10 \\
10\end{array}$ & $\begin{array}{l}\bar{\sigma} \\
\text { مे } \\
\text { مे } \\
\text { மn }\end{array}$ & $\begin{array}{l}\stackrel{0}{\overrightarrow{2}} \\
\stackrel{2}{\vec{n}} \\
\stackrel{10}{n}\end{array}$ & 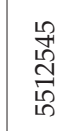 \\
\hline $\begin{array}{l}\vec{z} \\
0 \\
0 \\
0 \\
u\end{array}$ & 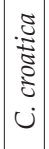 & 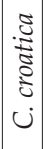 & 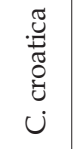 & 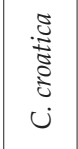 & 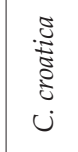 & 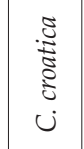 & 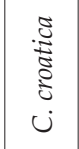 & 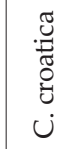 & 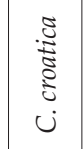 & 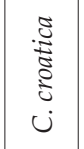 & 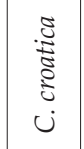 & 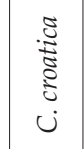 & $\begin{array}{l}\text { J } \\
\stackrel{0}{0} \\
0 \\
0 \\
ن\end{array}$ & 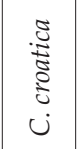 & $\begin{array}{l}\tilde{\Xi} \\
\tilde{\Xi} \\
0 \\
0 \\
ن \\
ن\end{array}$ & 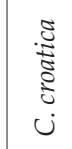 \\
\hline 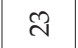 & $\mathbb{N}$ & $\stackrel{\mathscr{2}}{\sim}$ & $\sim$ & సิ & $\stackrel{\sim}{\sim}$ & ৯े & లి & $\vec{m}$ & กี & $m$ & के & $\stackrel{\mathscr{m}}{\mathrm{m}}$ & లి & के & $\stackrel{\infty}{\infty}$ & ले \\
\hline
\end{tabular}


lutea L. ssp. symphyandra (Murb.) Hayek, Ligusticum lucidum Mill., Solidago virgaurea L., Carex brachystachys Schrank, Calamagrostis sp., Hieracium sp., Clematis alpina (L.) Mill., Erica carnea L., Daphne alpina L., Juniperus communis L. ssp. alpina (Sm.) Celak., Salix appendiculata Vill., Rhododendron hirsutum L.

\section{Napa (Mt Obruč)}

Napa is a rocky peak on Mt Obruč located in the Pakleno area near a trail leading to the Hahlić mountain lodge. Several plants of A. kitaibelii grow from the fissures in limestone exposed to the east. Plant species that grow together with A. kitaibelii are Asplenium ruta-muraria, A. trichomanes L., Erigeron glabratus Bluff et Fingerh., Micromeria thymifolia (Scop.) Fritsch, Leontopodium alpinum Cass., Silene saxifraga L., Campanula cespitosa Scop., Calamagrostis sp., Hieracium sp., Juniperus communis ssp. alpina, Daphne alpina, Salix appendiculata.

\section{Belvedere near Špilja in the Pakleno area (Mt Obruč)}

A few hundred meters from the Napa Peak and near the Špilja locality in the Pakleno area there is an unnamed lookout point located on the left side of the trail (in the direction of the Hahlić mountain lodge). Several dozen plants of Aquilegia kitaibelii grow from fissures in vertical limestone rock exposed to the east. Plant species that grow together with A. kitaibelii are Asplenium ruta-muraria, Campanula cochleariifolia Lam., Micromeria thymifolia, Carex brachystacys, Sesleria tenuifolia Schrad., Clematis alpina, Teucrium montanum L., Juniperus communis ssp. alpina, Daphne alpina.

\section{Crni vrh}

Crni vrh (1335 m a.s.l.) is the highest peak in the eastern section of the Mt Obruč group and it is located several kilometers from the winter resort of Platak. Several dozen plants of $A$. kitaibelii grow from fissures in limestone exposed to the east and northeast. Plant species that grow together with A. kitaibelii are Campanula justiniana Witasek, Carex brachystachys, Galium lucidum, Genista sericea Wulfen, Micromeria thymifolia, Athamanta turbith (L.) Brot. ssp. haynaldii (Borbás et Euchtr.) Tutin., Sesleria tenuifolia, Hieracium sp., Satureja subspicata Bartl. ex Vis. ssp. liburnica Šilić.

Aquilegia kitaibelii was also found in two new locations in the central section of $\mathrm{Mt}$ Velebit and in fourteen new localities in the southern section of Mt Velebit. The presence of A. kitaibelii in the southern part of Mt Velebit (Paklenica National Park, Buljma, Babin vrh, Crljeni kuk, Kom, Komić, Malovan, Segestin, Sveto Brdo, Tulove grede) was mentioned by Degen (1938), Alegro (2004) and Nikolić (ed.) (2014). In general, A. kitaibelii grows on rocks together with other petrophilous plants.

\section{Gornje Branjevine 1}

Almost at the end of the Gornje Branjevine grassland, there is a group of Picea abies (L.) H. Karst trees. Several dozen plants of Aquilegia kitaibelii grows near the Premužić Trail on limestone rocks with a north - northwestern exposure, together with Cardaminopsis croatica, Sedum sexangulare L., Saxifraga paniculata Mill., Edraianthus tenuifolius (Waldst. et Kit.) A. DC., Campanula waldsteiniana Shult., Arenaria gracilis Waldst. et Kit., Athamanta turbith, Inula ensifolia L., Sesleria tenuifolia, Helianthemum nummularium (L.) Mill. ssp. obscurum (Čelak.) Holub, Thymus longicaulis C. Presl, Juniperus communis ssp. alpina, Satureja subspicata, Arctostaphylos uva-ursi (L.) Spreng., Rosa spinossisima L., Amelanchier ovalis Medik., Abies alba, Picea abies. 


\section{Below Ograđenica}

Several dozen plants of $A$. kitaibelii grow near the Premužić Trail, and near the crossing for the Ograđenica Mountain Shelter in the central section of Mt Velebit. Aquilegia kitaibelii grows on limestone rocks on both sides of the trail and is exposed to the west. Habitats are partly in the shadow of beech forests. Plant species that grow together with A. kitaibelii are Cardaminopsis croatica, Asplenium ruta-muraria, Micromeria thymifolia, Moehringia muscosa L., Corydalis ochroleuca Koch., Arabis turrita L., Valeriana tripteris L., Thalictrum aquilegifolium L., Solidago virgaurea, Galium mollugo L., Athamantha turbith ssp. haynaldii, Dianthus integer Vis., Ligusticum lucidum, Melica nutans L., Rosa sp., Daphne alpina, Juniperus communis ssp. alpina, Cotoneaster nebrodensis (Guss.) K. Koch, Populus tremula L., Sorbus austraca (Beck) Hedl., Fagus sylvatica L., Picea abies.

\section{Rujanska kosa}

Rujanska kosa is a meadow strewn with stones and located between Veliko Rujno and Malo Rujno. Aquilegia kitaibelii grows on rocks exposed to the southeast and southwest. Plant species that grow together with A. kitaibelii are Thalictrum minus L., T. aquilegifolium, Asperula sp., Cornus mas L., Viburnum lantana (L.) Spreng, Pinus nigra Arnold.

\section{Milovačka gora}

Milovačka gora is a rocky area located near the trail from Veliko Rujno to Bojinac. Aquilegia kitaibelii grows on rocks exposed to the northwest and northeast. Plant species that grow together with A. kitaibelii are Genista sylvestris Scop. ssp. dalmatica (Bartl.) H. Lindb, Globularia cordifolia L., Teucrium chamaedrys L., Cotoneaster nebrodensis, Frangula rupestris (Scop.) Schur, Ostrya carpinifolia Scop., Fagus sylvatica.

\section{Bojinac}

Bojinac is a rocky area located in the western part of Paklenica National Park. Aquilegia kitaibelii grows from the fissures in limestone exposed to the northwest and northeast. Plant species that grow together with A. kitaibelii are Polygala nicaensis Risso ex W. D. J. Koch, Micromeria thymifolia, M. croatica (Pers.) Schott, Campanula waldsteiniana, C. pyramidalis L., Teucrium montanum, Amelanchier ovalis, Frangula rupestris, Cotoneaster nebrodensis, Ostrya carpinifolia, Fagus sylvatica.

\section{0. Žlibati kuk}

Žlibati kuk is a peak (799 m a.s.l.) located above Veliki Vaganac in Paklenica National Park. Aquilegia kitaibelii grows on scree exposed to the northeast and northwest. Plant species that grow together with it are Micromeria croatica, Campanula waldsteiniana, $C$. pyramidalis, Tulipa sylvestris L., Cotoneaster nebrodensis, Amelanchier ovalis, Frangula rupestris, Ostrya carpinifolia, Fraxinus ornus L.

\section{Bilig}

Bilig is a peak in the western part of Paklenica National Park. Plants of A. kitaibelii grow on rocks and scree exposed to the southwest and southeast. Additionally, the habitat is exposed to strong northerly winds. Plant species that grow together with $\mathrm{A}$. $\mathrm{ki}$ taibelii are Tulipa sylvestris, Globularia cordifolia, Cotoneaster nebrodensis, Amelanchier ovalis, Ostrya carpinifolia.

\section{Zupkov dočić}

Zupkov dočić is located below Veliki Golić Peak (1236 m a.s.l.) in Paklenica National Park. Aquilegia kitaibelii was found on rocks exposed to the southwest, and located at the 
edge of a black pine forest. Plant species that grow together with A. kitaibelii are Campanula waldsteiniana, C. pyramidalis, C. velebitica Borbas, Micromeria croatica, Cotoneaster nebrodensis, Teucrium chamaedrys, Globularia cordifolia, Genista sylvestris ssp. dalmatica, Frangula rupestris, Amelanchier ovalis, Pinus nigra.

\section{Zanzibar}

Zanzibar is a spring between Veliko Rujno and mountain hut Paklenica. A large population of $A$. kitaibelii was found inside a beech forest on rocks exposed to the southeast. Plant species that grow together with A. kitaibelii are Polygala vulgaris L., Knautia drymeia Heuff., Arabis hirsuta (L.) Scop., Campanula glomerata L., Silene vulgaris (Moench.) Garcke, Helianthemum nummularium (L.) Mill. ssp. glabrum (Koch) Wilczek, Chamaecytisus hirsutus (L.) Briq., Acer pseudoplatanus L., A. obtusatum Kit., Fraxinus ornus, Fagus sylvatica.

\section{Below Buljma}

A large population of $A$. kitaibelii was found below Buljma Peak (1415 m a.s.l.) where it grows on rocks exposed to the southwest. Plant species that grow together with $A$. kitaibelii are Asplenium trichomanes, A. ceterach L., Silene vulgaris, Scrophularia canina L., S. heterophylla Willd. ssp. laciniata (Waldst. et Kit.) Maire et Petitm., Sesleria tenuifolia, Solidago virgaurea, Globularia cordifolia, Amelanchier ovalis, Sorbus aria (L.) Crantz, Ostrya carpinifolia, Fagus sylvatica.

\section{Below Rapavac}

Rapavac (1617 m a.s.l.) is a well-known peak in Paklenica National Park. Aquilegia kitaibelii grows on rocks located between a subalpine beech forest and a Pinus mugo forest. Plants of A. kitaibelii are exposed to the southwest and grow together with Campanula waldsteiniana, C. velebitica, Cerastium dinaricum G. Beck et Szyszył, Achillea clavenae L., Drypis spinosa L., Paronychia kapela (Hacq.) A. Kerner., Rhamnus alpinus L. ssp. fallax (Boiss.) Maire et Petitm., Pinus mugo Turra, Fagus sylvatica.

\section{Lipa staza Trail}

The Lipa staza Trail is a part of the trail from Babin Kuk (1431 m a.s.l.) to Vaganski Vrh peak (1757 m a.s.l.) in Paklenica National Park. Aquilegia kitaibelii grows on rocks exposed to the south, southeast and southwest together with Campanula waldsteiniana, C. velebitica, Cerastium dinaricum, Linaria alpina L., Achillea clavenae, Bunium alpinum Waldst. et Kit., Iberis pruitii Tineo, Paronychia kapela, Biscutella laevigata L., Sesleria tenuifolia, Drypis spinosa, Rhamnus alpinus ssp. fallax, Pinus mugo, Fagus sylvatica.

\section{Below Malovan}

The presence of A. kitaibelii on Malovan Peak was reported by Degen (1938). During the present investigation A. kitaibelii was also found below Malovan where it grows on rocks exposed to the northeast and northwest. Plant species that grow together with $A$. kitaibelii are Asplenium trichomanes, A. ruta-muraria, A. adiantum-nigrum L., Primula kitaibeliana Schott., Cerastium dinaricum, Astrantia major L., Buphthalmum salicifolium L., Lamium galeobdolon (L.) L., Heracleum sphondylium L., Dryas octopetala L., Globularia cordifolia, Cotoneaster integerrimus Medik., Amelanchier ovalis, Pinus mugo, Fagus sylvatica.

\section{Zala Ploča}

Zala Ploča is a rocky area between Čičina Dolina and Malovan. Aquilegia kitaibelii grows from fissures in limestone exposed to the east and southeast. Plant species that 
grow together with $A$. kitaibelii are Asplenium ruta-muraria, A. trichomanes, A. adiantumnigrum, Bunium alpinum, Cerastium dinaricum, Heracleum sphondylium, Iberis pruitii, Linaria alpina, Paronychia kapela, Androsace villosa L., Teucrium montanum, Globularia cordifolia, Pinus mugo.

\section{9. Čičina Dolina}

Čičina Dolina is a small pass in the southern section of Mt Velebit. Aquilegia kitaibelii grows on rocks exposed to the south, southeast and southwest. Plant species that grow together with A. kitaibelii are Globularia cordifolia, Teucrium montanum, Campanula velebitica, Asplenium adiantum-nigrum, A. ruta-muraria, A. trichomanes, Cerastium dinaricum, Iberis pruitii, Paronychia kapela.

\section{Bukva Crossing}

Bukva Crossing is a place where the trails leading to Tulove Grede and Veliko Libinje cross. Aquilegia kitaibelii was found on rocks nearby the gravel road, where it grows with Asplenium trichomanes, A. ruta-muraria, A. adiantum-nigrum, Campanula waldsteiniana, C. velebitica, Cerastium dinaricum, Solidago virgaurea, Paronychia kapela, Sesleria tenuifolia, Globularia cordifolia, Rhamnus alpinus ssp. fallax.

\section{Localities of Cardaminopsis croatica}

New localities of Cardaminopsis croatica were found in the Gacka Region and on Mt Velebit. During this research, C. croatica was not found on Mt Risnjak although this locality was mentioned by ŠEGULJA et al. (1994).

\section{Obilje 1}

Obilje is a part of the settlement of Prozor near the town of Otočac in the Gacka Region. Several plants of Cardaminopsis croatica grow near a gravel road in an Abies alba forest on limestone rock exposed to the south. Plant species that grow together with $C$. croatica are Asplenium trichomanes, A. ruta-muraria, Moehringia muscosa, Corydalis ochroleuca, Cardamine impatiens L., Geranium robertianum L., Sedum telephium L. ssp. maximum (L.) Krock., Mycelis muralis (L.) Dumort., Calamintha grandiflora (L.) Moench, Fragaria vesca L., Lamium galeobdolon, Lamium orvala L., Mercurialis perennis L., Stellaria holostea L., Lilium martagon L., Carex sylvatica Huds., Teucrium chamaedrys, Clematis vitalba L., Lonicera xylosteum L., Euonymus verrucosa Scop., Euonymus latifolia (L.) Mill., Corylus avellana, Fraxinus ornus, Sorbus torminalis (L.) Crantz.

\section{Obilje 2}

Limestone rock situated several dozen meters from a gravel road and surrounded by Carpinus betulus L., Quercus petraea (Matt.) Liebl. and an Abies alba forest is another new locality of $C$. croatica. Plant species that grow together with C. croatica are Polypodium vulgare L., Moehringia muscosa, Corydalis ochroleuca, Cardamine impatiens, Euphorbia amygdaloides L., Asarum europaeum L., Geranium robertianum, Lamium galeobdolon, L. orvala, Lathyrus vernus (L.) Bernh., Polygonatum multiflorum (L.) All., Potentilla micrantha Ramond ex DC., Stellaria holostea, Veronica chamaedrys L., Tanacetum corymbosum (L.) Sch. Bip. ssp. corymbosum, Carex pilosa Scop., Lonicera xylosteum, Cornus mas, Corylus avellana, Ligustrum vulgare L., Viburnum lantana, Staphylea pinnata L., Pyrus pyraster Burgsd., Fraxinus ornus, Fagus sylvatica. 


\section{Ličko Lešće}

In Ličko Lešće there is a well-known center for the reintroduction of indigenous fish. The center is surrounded by limestone rocks with different exposures. Several plants of Cardaminopsis croatica was found on several rocks exposed to the northeast together with Asplenium ceterach, A. ruta-muraria, A. trichomanes, Polypodium vulgare, Corydalis ochroleuca, Erophila praecox (L.) DC. ssp. praecox (Steven) Walp., Saxifraga rotundifolia L., Geranium macrorrhizum L., G. robertianum, G. lucidum L., Sedum album L., S. telephium ssp. maximum, S. sexangulare, Campanula pyramidalis, Arabis turrita, Mycelis muralis, Potentilla micrantha, Pimpinella saxifraga L., Lamium maculatum L., Melica ciliata L., Stachys recta L., Poa sp., Clematis vitalba, Rhamnus cathartica L., Ligustrum vulgare, Viburnum lantana.

\section{Crno Jezero}

Crno Jezero is a settlement situated on the southern edge of the Gacka Region. A new locality of C. croatica was found on the outskirts of the settlement (in the direction of Kuterevo) in a forest of Fagus sylvatica and Abies alba. Several dozen meters from the road that connects Otočac and Kuterevo there is a limestone rock exposed to the southeast. Plant species that grow together with several plants of $C$. croatica are Asplenium trichomanes, Asplenium scolopendrium L., Moehringia muscosa, Corydalis ochroleuca, Geranium macrorrhizum, G. robertianum, Pulmonaria officinalis L., Melica ciliata, Solanum dulcamara L., Clematis vitalba, Euonymus verrucosa, Staphylea pinnata, Crataegus monogyna Jacq. emend. Lindm., Malus sylvestris Mill., Tilia platyphyllos Scop., Acer obtusatum, Ostrya carpinifolia.

\section{Buljma}

Buljma Peak (1421 m a.s.l.) is situated in the central section of Mt Velebit, about 25 minutes by foot from the Alan mountain lodge. A new locality of $C$. croatica is situated on the south slopes of Buljma Peak. Several dozen plants of C. croatica grow near the Premužić Trail on limestone rocks with a western-southwestern exposure. Plant species that grow together with C. croatica are Arenaria gracilis, Campanula waldsteiniana, Inula hirta L., Dianthus sp., Pimpinella sp., Sesleria tenuifolia, Thymus acicularis Waldst. et Kit., Globularia meridionalis (Podp.) Schwarz, Satureja subspicata, Rubus idaeus L., Genista sericea, Juniperus communis ssp. alpina, J. sabina L., Daphne alpina, Frangula rupestris (Scop.) Schur, Rosa spinosissima.

\section{Border of North Velebit National park}

A new locality of C. croatica was found in Velebit Nature Park, about $100 \mathrm{~m}$ from the border of North Velebit National park. Several C. croatica plants grow near the Premužić Trail on limestone rocks with a western-southwestern exposure. Plant species that grow together with C. croatica are Campanula waldsteiniana, Libanotis nitida Vis., Laserpitium siler L., Sesleria tenuifolia, Helianthemum canum (L.) Baumg., Thymus acicularis, Globularia meridionalis, Genista sericea, Satureja subspicata, Daphne alpina, Amelanchier ovalis.

\section{Gornje Branjevine 2}

Another locality of C. croatica is found in a Fagus sylvatica forest where it grows on limestone rocks with a western-southwestern exposure. Plant species that grow together with C. croatica are Asplenium trichomanes, Sedum ochroleucum Chaix, Mycelis muralis, Arabis turrita, Aremonia agrimonoides (L.) DC., Centaurea triumfetti All., Prenanthes purpurea, Pseudolysimachion spicatum (L.) Opiz, Geranium robertianum, Rubus sp., Sorbus aria, Fagus sylvatica, Acer pseudoplatanus. 


\section{Gornje Branjevine 1}

A decription of this locality is given above (see locality number 5: Gornje Branjevine $1)$.

\section{Gornje Branjevine 3}

Cardaminopsis croatica was also found on another group of limestone rocks near the Premužić Trail. Plant species that grow together with C. croatica are Asplenium rutamuraria, Arabis turrita, Corydalis ochroleuca, Buphthalmum salicifolium, Mercurialis perennis, Mycelis muralis, Galium mollugo, Geranium robertianum, Scrophularia heterophylla ssp. laciniata, Heracleum sphondylium, Sesleria tenuifolia, Rubus idaeus, Satureja montana L., Fagus sylvatica.

\section{Below Ograđenica}

A description of this locality is given above (see locality number 6: Below Ograđenica).

\section{Premužić Trail between Ograđenica and Korita Spring}

Another locality of C. croatica was noted between the crossing to the Ograđenica mountain shelter and Korita Spring. Plants of C. croatica grow near the Premužić Trail on limestone rocks with a southern-southwestern exposure. Plant species that grow together with C. croatica are Asplenium trichomanes, A. ruta-muraria, Micromeria thymifolia, Arabis turrita, Dianthus petraeus Waldst. et Kit. ssp. petraeus, Geranium robertianum, Solidago virgaurea, Galium mollugo, Stellaria holostea, Poa sp., Satureja montana, Sorbus aria, Laburnum alpinum (Mill.) Bercht., Fagus sylvatica.

\section{Mliništa}

Near the Premužić Trail above the Mliništa grassland there is a huge limestone rock exposed to the northwest. The habitat of C. croatica is partly in the shadow of a Fagus sylvatica forest. Plant species that grow together with C. croatica are Cystopteris fragilis (L.) Bernh., Geranium robertianum, Moehringia muscosa, Arabis turrita, Campanula fenestrellata Feer, Saxifraga rotundifolia, Mycelis muralis, Corydalis ochroleuca, Actaea spicata L., Mercurialis perennis, Antriscus fumarioides (Waldst. et Kit.) Spreng., Fagus sylvatica.

About $500 \mathrm{~m}$ from the above mentioned rock there is another rock exposed to the south where C. croatica grows together with Asplenium trichomanes, Mycelis muralis, Moehringia muscosa, Campanula fenestrellata, C. pyramidalis, Arabis turrita, Geranium macrorrhizum, Euonymus latifolia, Ostrya carpinifolia, Acer obtusatum, Fagus sylvatica.

\section{Skorpovac}

About 100 m before the Skorpovac mountain shelter (in the direction of Dabarska Kosa or Baške Oštarije) there is another locality of C. croatica. Cardaminopsis croatica grows on rocks with a northeastern exposure together with Asplenium trichomanes, Polypodium vulgare, Dryopterys filix-mas (L.) Schott, Oxalis acetosella L., Geranium robertianum, Micromeria thymifolia, Mycelis muralis, Carduus acanthoides L., Calamintha grandiflora, Melittis melissophyllum L., Senecio ovatus (G. Gaertn., B. Mey. et Scherb.) Willd, Sesleria autumnalis (Scop.) F. W. Schultz, Rosa pendulina L., Lonicera xylosteum, Sorbus aria, Abies alba, Fagus sylvatica.

\section{Crossing of Premužić Trail and a timber dump}

About one hundred meters before the crossing of the Premužić Trail and a timber dump (in the direction of Dabarska Kosa or Baške Oštarije) there is a rocky area exposed 
to the south. Cardaminopsis croatica grows on rocks near the Premužić Trail and along the trail together with Asplenium trichomanes, A. ruta-muraria, Corydalis ochroleuca, Micromeria thymifolia, Mycelis muralis, Cnidium silaifolium (Jacq.) Simonk., Galium mollugo, Geranium robertianum, Solidago virgaurea, Sesleria autumnalis, Campanula pyramidalis, C. bononiensis, Satureja montana, Rosa pendulina, Viburnum lantana, Laburnum alpinum, Sorbus aria, Ostrya carpinifolia, Fraxinus ornus, Acer obtusatum, Fagus sylvatica.

Several hundred meters before this locality (in the direction of the Skorpovac mountain shelter) there is another rock in a Fagus sylvatica forest. Plant species that grow together with C. croatica are Asplenium trichomanes, Biscutella laevigata, Campanula fenestrellata, Campanula pyramidalis, Cardamine enneaphyllos (L.) Crantz, C. bulbifera (L.) Crantz, Moehringia muscosa, Geranium macrorrhizum, Saxifraga rotundifolia, Sesleria autumnalis, Euonymus latifolia, Acer obtusatum, A. pseudoplatanus, Ostrya carpinifolia, Fagus sylvatica.

\section{Crossing of Premužić Trail and a gravel road}

Some forty minutes by foot from the Skorpovac mountain shelter (in the direction of Dabarska Kosa or Baške Oštarije), the Premužić Trail crosses a gravel road. Several dozen meters before this crossing there is a group of limestone rocks with a northern-northeastern exposure. Plant species that grow together with C. croatica are Asplenium trichomanes, Polypodium vulgare, Geranium robertianum, Origanum vulgare L., Mycelis muralis, Hieracium sp., Prenanthes purpurea L., Sesleria autumnalis, Poa sp., Rosa pendulina, Acer pseudoplatanus, Ostrya carpinifolia, Acer obtusatum, Fagus sylvatica.

\section{Crossing to Budakovo Brdo}

Budakovo brdo (1317 $\mathrm{m}$ a.s.l.) is one of the well-known peaks in the central section of Mt Velebit. Cardaminopsis croatica was found near the Premužić Trail and before the crossing of Premužić Trail - Budakovo brdo (in the direction of Baške Oštarije). Cardaminopsis croatica grows on limestone rocks exposed to the south. Plant species that grow together with C. croatica are Asplenium trichomanes, Sedum sexangulare, Moehringia muscosa, Arabis turrita, Campanula pyramidalis, Sesleria autumnalis, Poa bulbosa L. var. vivipara (Koeler) Willd., Allium sp., Arenaria sp., Ostrya carpinifolia, Acer obtusatum, Fraxinus excelsior L.

\section{Dabarska kosa}

Another locality of $C$. croatica was situated about one kilometer from the beginning of Dabarska kosa (in the direction of the Skorpovac mountain shelter). Cardaminopsis croatica grows in an Ostrya carpinifolia forest on limestone rock exposed to the south. Plant species that grow together with C. croatica are Asplenium trichomanes, A. ceterach, Corydalis ochroleuca, Saxifraga tridactylites L., Sedum ochroelucum, Moehringia muscosa, Geranium robertianum, Sanguisorba minor Scop. ssp. muricata Briq., Arabis turrita, Allium fuscum Waldst. et Kit., Micromeria thymifolia, Melica ciliata, Asperula sp., Satureja montana, Prunus mahaleb L., Ostrya carpinifolia, Acer monspessulanum L., A. obtusatum, Fraxinus ornus.

Several hundred meters in the direction of the Skorpovac mountain shelter there is rock that looks like a table. Near this unusual rock, situated in an Ostrya carpinifolia forest, there is another locality of C. croatica. Cardaminopsis croatica grows on rock exposed to the south together with Asplenium ruta-muraria, Sedum ochroelucum, Moehringia muscosa, Geranium robertianum, Galium purpureum L., Micromeria thymifolia, Lamium maculatum, Mycelis muralis, Arabis turrita, Crocus vernus (L.) Hill, Valeriana officinalis L., Sesleria autumnalis, Melica uniflora Retz., Genista januensis Viv., Satureja montana, Acer monspessulanum, Fraxinus ornus. 


\section{Premužić Trail below Badanj 1}

Badanj (1164 m a.s.1.) is another well-known peak in the central section of Mt Velebit. Cardaminopsis croatica grows on rocks near the Premužić Trail as well as among rocks on the very trail. Plant species that grow together with C. croatica are Asplenium ruta-muraria, Corydalis ochroleuca, Campanula waldsteiniana, Arabis turrita, Sesleria autumnalis, Peltaria alliacea Jacq., Convallaria majalis L., Cruciata glabra (L.) Ehrend, Platanthera bifolia (L.) Rich., Iris graminea L., Valeriana tripteris, Carex flacca Schreb., Daphne mezereum L.

\section{Premužić Trail below Badanj 2}

Several dozen plants of $C$. croatica grow on limestone gravel along the gravel road in a Fagus sylvatica forest. Plant species that grow together with C. croatica are a mix of forest, ruderal and grassland species: Arenaria serpyllyfolia L., Moehringia trinervia (L.) Clairv., Clinopodium vulgare L., Plantago major L., Galium mollugo, Medicago lupulina L., Trifolium pratense L., Mycelis muralis, Aremonia agrimonioides, Prunella vulgaris L., Leucanthemum ircutianum (Turcz.) DC., Mercurialis perennis, Cardamine bulbifera, Scrophularia heterophylla ssp. laciniata, Acer pseudoplatanus, Stachys sylvatica L., Verbascum sp., Rubus idaeus, Satureja subspicata, Fagus sylvatica.

In conclusion, 20 new localities of Aquilegia kitaibelii and 19 new localities of Cardaminopsis croatica on the Dinaric Mountains in Croatia are described. The information gathered helps to broaden our knowledge of the population numbers, and environmental conditions of both these endemic species and on the distribution range of $C$. croatica. Additionally, A. kitaibelii is included in the list of NATURA 2000 Species. Accordingly, any information about new localities of $A$. kitaibelii is important because it enables a more reliable evaluation of the threat level, population numbers, and population health of this endemic species. The second species, C. croatica, is also a floristically interesting endemic, closely related to the preeminent plant model organism Arabidopsis thaliana. Our investigation has shown that neither of the studied chasmophytes (plants that grow in fissures in rock) is endangered at this moment. A potential threat could be the overgrowing of rock habitats by forest. However, because both species can exist in open, sunny habitats as well as in the shadow of forest, the degree of this threat is still rather low for now. Moreover, it was noticed that C. croatica is spreading on secondary, anthropogenic habitats on the edges of gravel roads. It is interesting to point out that the species were found together only at two localities on Mt Velebit. Once data on the many populations of $A$. kitaibelii and $C$. croatica are available, it will be possible to establish their ecological differentiation and the demarcation of the phytocenological communities where these species grow. Also, it will be possible to make more accurate conclusions about how they thrive within their area of natural distribution. This type of study can make valuable contributions to our knowledge about the NW Dinarides as an ancient refuge area rich in endemic and subendemic species. 


\section{REFERENCES}

Alegro, A. L., 2004: Plant world of NP “Paklenica” (In Croatian). In: Lukač, G. (ed.), Paklenički zbornik: Simpozij povodom 55. godišnjice NP “Paklenica”, Vol. 2., Uprava NP Paklenica, Starigrad Paklenica, p. 35-54.

Al-Shehbaz, I. A. \& O'Kane, Jr S. L., 2002: Taxonomy and phylogeny of Arabidopsis (Brassicaceae). The Arabidopsis Book doi:10.1199/tab.0001

Anonymous, 2009: Habitat types in Croatia (National habitat classification - NKS) (In Croatian). OfFICIal Gazette (OG) 119/09, 21-52.

Anonymous, 2013: Pravilnik o strogo zaštićenim vrstama (Ordinace of strictly protected species) (In Croatian). Official Gazette (OG) 144/13, 7-84.

Cullen, J. \& Heywood, V. H., 1964: Aquilegia L. In: Tutin, T. G., Heywood, V. H., Burges, N. A., ValentiNE, D. H. \& Moore, D. M. (eds.): Flora Europaea, Vol. 1, Cambridge University Press, Cambridge, p. 238-240.

Degen, A., 1938: Flora Velebitica. Vol. 2, p. 118-120; vol. 2, p. 165. Verlag der Ungar. Akademie der Wissenschaften, Budapest.

Domac, R., 1994: Croatian flora (In Croatian). Školska knjiga, Zagreb.

Fior, S., Li, M., Oxelman, B., Viola, R., Hodges, S. A., Ometto, L. \& Varotto, C., 2013: Spatiotemporal reconstruction of the Aquilegia rapid radiation through next-generation sequencing of rapidly evolving cpDNA regions. New Phytologist 198, 597-592.

Forenbacher, S., 1990: Mt Velebit and its flora (In Croatian). Školska knjiga, Zagreb.

Hohmann, N., Schmickl, R., Chiang, T-Y., Lučanová, M., Kolář, F., Marhold, K. \& Koch, M. A., 2014 : Taming the wild: resolving the gene pools of non-model Arabidopsis lineages. BMC Evolutionary Biology 14, 224-245.

Horvat, I., 1962: Vegetation of mountains in western Croatia (In Croatian). Prirodoslovna istraživanja JAZU, ser. Acta biologica 30, 65.

Horvat, I., Glavač, V. \& Ellenberg, H., 1974: Vegetation Suedosteuropas. GFV, Stuttgart.

Kremer, D., 2008: Aquilegia kitaibelii Schott. In: Skorup, V., Kovačić, S., Kremer, D. \& Mihelj, D., Velebit botanical garden - oasis at 1500 m (In Croatian). Javna ustanova "Nacionalni park Sjeverni Velebit", Krasno, p. 116-117.

Kremer, D., Lukač, G., Randić, M., Krušić, I., Kosalec, I. \& Ruščić, M., 2014: New localities of Berberis croatica Horvat and Teucrium arduini L. in the croatian Dinaric Mountains. Natura Croatica 23, 147-162.

KušAN, F., 1969: Vegetation cover of Mt. Biokovo (Flora and vegetation) (In Croatian). Prirodoslovna istraživanja JAZU, ser. Acta biologica 37, 5-224.

Nikolı́́, T. (ed.), 2014: Flora Croatica Database. Department of Botany, Faculty of Science, University of Zagreb. (http://hirc.botanic.hr/fcd). Prirodoslovno-matematički fakultet Sveučilišta u Zagrebu. [Last access: September 5, 2014]

Podobnik, A., Surina, B. \& Dakskobler, I., 2013: Story of Bertoloni columbine in Slovenia (In Slovenian). Proteus 75, 295-302.

Randić, M., 2010: Obruč (In Croatian). In: Nikolić, T., Topić, J. \& Vuković, N. (eds.), Botanički važna područja Hrvatske, Prirodoslovno-matematički fakultet i Školska knjiga, Zagreb, p. 295-331.

Schmickl, R., Paule, J., Klein, J., Marhold, K. \& Косн, M. A., 2012: The evolutionary history of the Arabidopsis arenosa complex: diverse tetraploids mask the Western Carpathian center of species and genetic diversity. PLoS ONE 7, e42691. doi:10.1371/journal.pone.0042691

SurINA, B., 2013: Heaths with dwarf ericaceous shrubs and Alpine juniper (Juniperus alpina) in the Dinaric Alps: A nomenclatorial and synsystematic re-appraisal. Acta Botanica Croatica 72, 113-132.

ŠEgulJA, N., 1994: Some peculiarity of vascular flora of National park Plitvička jezera (In Croatian). In: Trinajstić, I. (ed.), Zbornik radova: Simpozij - Pevalek, Flora i vegetacija Hrvatske, Sumarski fakultet Sveučilišta u Zagrebu, Koprivnica, p. 117-124.

ŠEgulja, N., LovašEn-Eberhardt, Ž., HršAK, V. \& LuKač, G., 1994: Review of the state of research of flora in National Park Risnjak (In Croatian). In: Frković, A. (ed.), Zbornik radova: 40 god. Nacionalnog parka Risnjak (1953-1993), Javno poduzeće Uprava nacionalnog parka Risnjak, Crni Lug, p. 71-77.

ŠILIĆ, Č., 1990: Endemic plants (In Bosnian). IP Svjetlost, Sarajevo, Beograd. 


\title{
SAŽETAK
}

\section{Novi lokaliteti endemičnih vrsta Aquilegia kitaibelii Schott i Cardaminopsis croatica (Schott, Nyman et Kotschy) Jáv. u Hrvatskoj}

\author{
D. Kremer, G. Lukač, A. Brkljačić, J. Brajković, K. Čulinović \& M. Randić
}

U razdoblju od svibnja 2010. do listopada 2014. godine na području sjeverozapadnih Dinarida u Hrvatskoj utvrđeni su novi, do sada u literaturi nezabilježeni lokaliteti dviju endemičnih, razmjerno rijetkih i floristički zanimljivih biljnih vrsta: Kitaibelova pakujca (Aquilegia kitaibelii Schott) i hrvatske gušarke (Cardaminopsis croatica (Schott, Nyman et Kotschy) Jáv.). U nas su obje vrste zakonom strogo zaštićene. Kitaibelov pakujac je rijetka, endemična vrsta rasprostranjena na području Hrvatske te Bosne i Hercegovine gdje raste u planinskim područjima na nadmorskoj visini između 1200 i $1700 \mathrm{~m}$. Noviji podaci navode prisutnost ove vrste i na planini Snežnik u Sloveniji. Kitaibelov pakujac je uvršten i na popis NATURA 2000 vrsta, dok je prema IUCN kategorizaciji označen kao DD (data deficient) vrsta. Kitaibelov pakujac se navodi i u Prilogu IV Direktive 92/43/EEZ o zaštiti prirodnih staništa i divljih biljnih i životinjskih vrsta. Kao strogo zaštićenu vrstu nalazimo ga i u Dodatku I Konvencije o zaštiti europskih divljih vrsta i priodnih staništa (tzv. Bernska konvencija). Zbog svega navedenog potrebno je nastaviti rad na prikupljanju svih relevantnih podataka o rasprostranjenosti i ekološkim zahtjevima ove vrste, brojnosti i stanju njenih prirodnih populacija, pa i u svrhu uspostave eventualnog monitoringa. Hrvatska gušarka također je endemična vrsta prirodno rasprostranjena u planinskim područjima Hrvatske i Bosne i Hercegovine na nadmorskoj visini između 500 i 1500 m. Prema novijim shvaćanjima vrsta je iz roda Cardaminopsis premještena u rod Arabidopsis. Činjenica da je hrvatska gušarka taksonomski bliska vrsti Arabidopsis thaliana, koja se kao biljka model koristi u brojnim znanstvenim istraživanjima, samo je dodatni razlog zašto je potrebno nastaviti prikupljanje svih dostupnih podataka i o ovoj vrsti.

Cilj rada je predstaviti po dvadesetak novih nalazišta Kitaibelova pakujca i hrvatske gušarke na području sjeverozapadnih Dinarida u Hrvatskoj.

Nova nalazišta Kitaibelova pakujca zabilježena su na širem području planinske skupine Obruča u zaleđu grada Rijeke te na području srednjeg i južnog Velebita. Do sada u literaturi nezabilježeni lokaliteti hrvatske gušarke utvrđeni su na području srednjeg Velebita i na padinama brežuljaka i brda u regiji Gackoj. Obje vrste na većini novih nalazišta rastu u pukotinama stijena zajedno s drugim petrofilnim vrstama, a Kitaibelov pakujac zabilježen je i na točilima. Zanimljivo je da su obje vrste zajedno na istom staništu utvrđene samo na lokalitetima Gornje Branjevine i podno Ograđenice uz Premužićevu stazu na području srednjeg Velebita. I jedna i druga vrsta oblikuju male, trenutno stabilne prirodne populacije na mjestima gdje je konkurentnost drugih vrsta znatno slabija. Za hrvatsku gušarku posebno su zanimljiva nalazišta u regiji Gackoj, gdje raste na termofilnim stijenama različitih ekspozicija, u okruženju mješavine termofilnih submediteranskih vrsta i pojedinih brdskih vrsta bukovo-jelovih šuma. Uz Premužićevu stazu na srednjem Velebitu hrvatska gušarka se također pojavljuje u nekim termofilnim zajednicama šumskih stijena. Opaženo je da, osim stijena, hrvatska gušarka nastanjuje i neka sekundarna, antropogena staništa, kao što su rubovi šumskih cesta, što ukazuje na ekološku plastičnost ove endemične vrste sjeverozapadnih Dinarida.

Jednom kada budu poznati podatci o većem broju populacija ovih dviju vrsta biti će moguće napraviti i fitocenološku karakterizaciju biljnih zajednica u kojima se one pojavljuju. Isto tako, biti će moguće dati preciznije prijedloge o njihovoj in situ zaštiti, ali i o eventualnoj potrebi za poduzimanjem mjera ex situ zaštite. Ovo istraživanje predstavlja i doprinos poznavanju biljnog svijeta sjeverozapadnih Dinarida u Hrvatskoj, području poznatom po više endemičnih i subendemičnih vrsta. 